\title{
HyP-ABC: A Novel Automated Hyper-Parameter Tuning Algorithm Using Evolutionary Optimization
}

This paper was downloaded from TechRxiv (https://www.techrxiv.org).

\section{LICENSE}

CC BY-SA 4.0

SUBMISSION DATE / POSTED DATE

01-06-2021 / 12-11-2021

\section{CITATION}

Zahedi, Leila; Mohammadi, Farid Ghareh; Amini, M. Hadi (2021): HyP-ABC: A Novel Automated HyperParameter Tuning Algorithm Using Evolutionary Optimization. TechRxiv. Preprint. https://doi.org/10.36227/techrxiv.14714508.v3

$\mathrm{DOI}$ 


\title{
HyP-ABC: A Novel Automated Hyper-Parameter Tuning Algorithm Using Evolutionary Optimization
}

\author{
Leila Zahedi, Farid Ghareh Mohammadi, and M. Hadi Amini
}

\begin{abstract}
Machine learning techniques lend themselves as promising decision-making and analytic tools in a wide range of applications. Different ML algorithms have various hyperparameters. In order to tailor an ML model towards a specific application working at its best, its hyper-parameters should be tuned. Tuning the hyper-parameters directly affects the performance. However, for large-scale search spaces, efficiently exploring the ample number of combinations of hyperparameters is computationally expensive. Many of the automated hyper-parameter tuning techniques suffer from low convergence rates and high experimental time complexities. In this paper, we propose $\mathrm{HyP}-\mathrm{ABC}$, an automatic innovative hybrid hyperparameter optimization algorithm using the modified artificial bee colony approach, to measure the classification accuracy of three ML algorithms: random forest, extreme gradient boosting, and support vector machine. In order to ensure the robustness of the proposed method, the algorithm takes a wide range of feasible hyper-parameter values and is tested using a realworld educational dataset. Experimental results show that HyP$\mathrm{ABC}$ is competitive with state-of-the-art techniques. Also, it has fewer hyper-parameters to be tuned than other population-based algorithms, making it worthwhile for real-world HPO problems.
\end{abstract}

Impact Statement-Hyper-parameter tuning is an essential step in automated machine learning, and it reduces the burden of manual tasks and is economical and time-efficient. However, searching through an ample space (wide ranges) of hyperparameter is a time-consuming task. This study presents a modified evolutionary optimization algorithm explicitly developed for hyper-parameter tuning in machine learning algorithms as an alternative to other methods to partly overcome the limitations mentioned above. The proposed algorithm is tested on an educational dataset for a binary classification problem. It shows its competitiveness compared to other existing methods and is ready to support users in various other applications.

Index Terms-Hyper-parameter Tuning, Machine Learning Optimization, AutoML, Artificial Bee Colony Algorithm, Education

\section{INTRODUCTION}

\section{A. Motivation}

D eploying Machine Learning (ML) for real-world problems causes challenges, such as selecting the proper model among a set of candidate models, hyper-parameter tuning, or selecting the best features to feed to the ML models. An ML model's performance depends on such initial design

Submitted November 10, 2021

Leila Zahedi is with Knight Foundation School of Computing and Information Sciences Florida International University, Miami, FL 33199 USA (e-mail: lzahe001@ fiu.edu).

Farid Ghareh Mohammadi is with Department of Computer Science at University of Georgia, Athens, GA 77005 USA. (e-mail: farid.ghm@uga.edu).

M. Hadi Amini is with Knight Foundation School of Computing and Information Sciences Florida International University, Miami, FL 33199 USA (e-mail: amini@cs.fiu.edu). decisions, which can be confusing to new users who desire to choose the most appropriate model [1]. These decisions are usually made based on the model's obtained quality or in other words Performance Indicator (PI) [2]. According to the principle of Occam's razor, a model should not be too simple nor too complex so that it can be efficient and can also capture data patterns without overfitting [1], [3]. Hyper-parameter tuning chooses the values that have a more considerable impact on the ML model's performance. Hyperparameters on an ML model control the convergence of the learning process. Hence, it is crucial to find the optimal values for their hyper-parameters [4]. Independent from the expertise level of the users, obtaining desirable results using different datasets can be conducted manually and is a tedious task [5], [6]. This is where Automated ML (AutoML) comes into the picture to alleviate such burdens. Automated Hyper-parameter Optimization (HPO) is one of the decisive and primary tasks of AutoML [7].

Bhattacharyya et al., in [8] proposed an automated heartbeat classification framework. This framework takes advantage of an exhaustive HPO method, Grid Search, to tune the hyperparameters of random forest and support vector machine classifiers. The results showed significant enhancement of efficiency. In another study [9], Vu et al., proposed a quantitative and constraint preserving score to ease the process of choosing hyper-parameters of visualization algorithms. This method uses Bayesian optimization to search the search space and obtain the most appropriate configuration. The experimental results shows promising results as well as users' interest.

In HPO, the problem is more challenging when there are so many configurations; as hyper-parameter value ranges increase, the search space grows exponentially. Therefore, common practices of tuning hyper-parameters for large search spaces is not ideal . In this work, we will show how populationbased algorithms (PBAs) can tune the hyper-parameters of different ML algorithms.

\section{B. Objective}

Manual search or automated exhaustive search among $n^{k}$ configurations of hyper-parameters are impractical and time inefficient [10]. Manual tuning does not provide reproducibility. Exhaustive search, on the other hand, suffers from dimensionality issues in large search spaces. As such, there has been increased research in HPO to optimize the performance of ML models regarding both accuracy and time.

HPO methods may change the final model in comparison to when a model is being trained with default hyper-parameters [11]. Hence, they provide fairness to research and scientific 


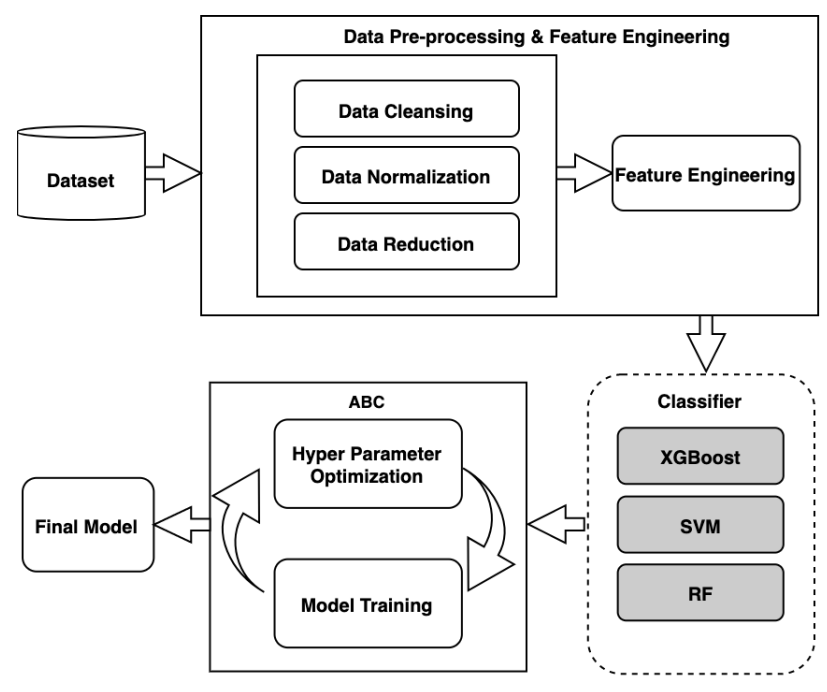

Fig. 1: General framework of the study

studies. Also, when making decisions, time is of the essence. Hence, many black-box optimization models do not fit such optimization problems because they do not consider the function evaluation time [6]. Therefore, proper algorithms should be applied to such problems to find the optimal set of hyperparameters. Mohammadi et al. provided different examples of applying PBAs, such as feature extraction, in different domains and encouraged researchers to apply such methods to solve large-scale optimization problems [12]. Our goal is to design and develop an HPO framework to tackle the challenges discussed above; The framework utilizes $\mathrm{ABC}$ to minimize the run-time caused by large dimensions of search-space scales, known as Curse of Dimensionality (CoD), and possibly improve the accuracy of the ML model. This framework will assist the predictions for intelligent and real-time decisionmaking and lessen the financial costs and labor burden.

In the literature, $\mathrm{ABC}$ has been used to tune the hyperparameters of some deep learning algorithms, convolutional neural networks, and least squares support vector machine [13]-[15]. To fit ABC to HPO problems of ML algorithms, this paper uses HyP-ABC to build a binary classification model. Figure 1 shows the general diagram of our experiment. We utilize HyP-ABC to tune the main hyper-parameters of Random Forest (RF), Extreme Gradient Boosting (XGBoost), and Support Vector Machine (SVM). These three models are selected based on the reports from an earlier study [11] indicating the high tuning-time using some other automated HPO approaches.

\section{Contribution}

The main contributions of this study are as follows:

1) This paper develops a novel optimization method that fits HPO problems for three ML algorithms using a modified evolutionary optimization algorithm.

2) The HyP-ABC algorithm outperforms the HPO methods utilized in an existing study in 2021 [11] in terms of both tuning time and accuracy.
3) The HyP-ABC algorithm is competitive with other stateof-the-art existing HPO methods.

4) This paper is the first to explore the optimization of ML models tailored towards large-scale educational datasets like MIDFIELD.

\section{Organization of Paper}

The rest of the paper is organized as follows: in section II we provide an introduction to PBA and a comprehensive explanation of $\mathrm{ABC}$. Then, we present the related work regarding hyper-parameter tuning and different automated tuning methods in section III. We also cover ABC applications in this section. Section IV covers hyper-parameter tuning using the $\mathrm{ABC}$ approach and the advantages of $\mathrm{ABC}$ over other techniques. In section $\mathrm{V}$, we propose the $\mathrm{HyP}-\mathrm{ABC}$ algorithm; an ABC-based algorithm fitted to HPO problems. The experimental methodology is presented in Section VI, and Section VII presents experimental results to demonstrate the performance achievable by the proposed approach. Last, section IX concludes the paper and discusses future directions.

\section{Population-Based Optimization Algorithms}

Population-based optimization algorithms work based on generating and updating individuals in each generation; this continues until the optimal or close to the optimal solution is identified and or until the stopping criterion. [16]. These algorithms are based on the interaction between different individuals to find acceptable quality solutions. These algorithms garnered much attention because of their excellent performance and are particularly useful to solve optimization problems [17]. PBAs include different heuristic algorithms such as Genetic Algorithms (GAs) [18], Particle Swarm Optimization (PSO) [19], Ant Colony Optimization (ACO) [20], Grey Wolf Optimization (GWO) [21], Fish Swarm Algorithms (FSA) [22], and Artificial Bee Colony (ABC) [23]. The main difference between these algorithms is how a population is generated, and selected [24]. One of the main advantages of these algorithms is that, unlike many model-based optimization methods, they have the capability of parallelization [25]. Among these algorithms, $\mathrm{ABC}$ is one of the most popular methods due to the excellent exploration and exploitation capabilities to find the satisfactory solution [26]. Therefore, $\mathrm{ABC}$ was chosen for further exploration in this study.

\section{A. Artificial Bee Colony (ABC)}

$\mathrm{ABC}$, first defined by Karaboga [27], is one of the most recent and popular SI algorithms that simulate honey bees' foraging behavior. In $\mathrm{ABC}$, different groups of bees fly around in an area (search space). The bee colony is divided into: 1) employed, 2) onlookers, and 3) scouts. At the initialization stage, a random set of food sources get selected by scout bee, and their amount of nectar is determined. Then scout bees share the nectar information with employed bees and employed bees visit those food sources and choose a new food source in the vicinity and compare it with the other food source and select the better one. Next, the information is 
shared with onlooker bees, and they may or may not select a food source according to the information received (nectar amount). The higher the amount of nectar, the higher is the chance of getting selected by onlooker bees. If selected, the onlooker bee chooses a new food source in that neighborhood and compares the two food sources, and the preferred food source gets selected, and if not selected, the onlooker bee moves toward the next food source for evaluation. Employed and onlooker bees' responsibility is exploration based on their own experience, where they leave poor food sources and move toward better ones through a greedy selection process. When a food source exploitation is exhausted or can not be further improved (based on trial limits), the employed bee discards the food source and becomes a scout bee and start exploration where it selects a new random food source and replaces it with the abandoned food source.The pseudo-code of the original $\mathrm{ABC}$ is described in Algorithm1 [28].

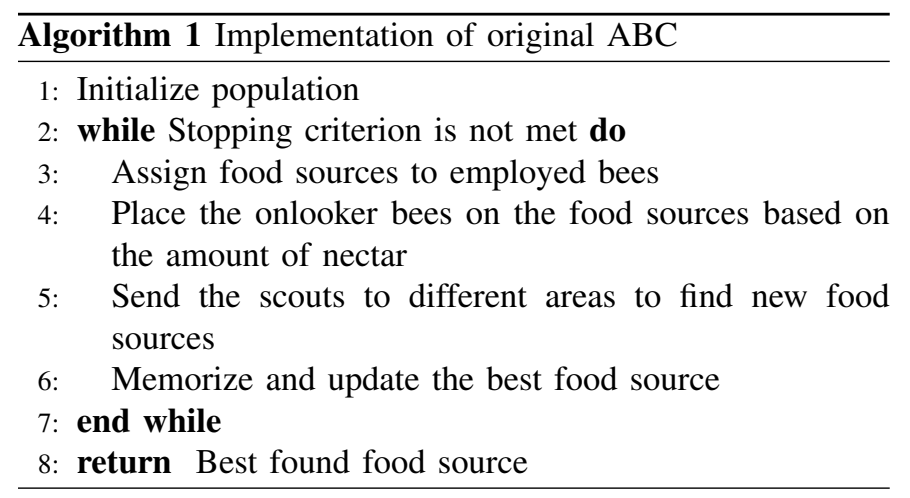

\section{RELATED WORKS}

\section{A. Hyper-parameter Tuning}

Grid Search (GS) is a brute-force method that searches all the hyper-parameters within a fixed search space. The advantage of this method is that it obtains the optimal solution in a discrete search space [11]. However, it is computationally expensive in large-scale spaces. Random Search (RS) is another approach that randomly selects the values with limited resources (time or number of iterations). This method works well for search spaces containing continuous values. However, luck plays a part in this method, and giving more resources increases the chance of getting better results [11]. There are some improved versions of RS, such as Hyperband (HB) which is more efficient especially in cases where resources are limited.

Unlike GS and RS, Bayesian Optimization (BO) [29] prevents evaluating many of the unnecessary configurations based on the evaluations of the previous steps. BO is an approach that is commonly used for HPO problems and, unlike model-free approaches such as GS and RS, is a model-based technique. In other words, the future evaluations are based on the previously evaluated points. However, since BO methods work sequentially to balance exploitation and exploration a, they are challenging to parallelize. The most popular BO techniques in HPO problems are Gaussian Processes (GP) and Tree-structured Parzen Estimator (TPE).
GA and PSO are the popular population-based algorithms used in HPO problems. GA is a search strategy inspired by Charles Darwin's theory of natural evolution. It is based on the process of natural selection, where the fittest individuals are the ones being selected for the next steps of reproduction to produce next generation offspring. PSO is another evolutionary algorithm that traverses the search space in a semi-random manner. This algorithm is inspired by individual and social behaviors of biological populations [24].

For some models, such as tree-based models ( $\mathrm{RF}$ and XGBoost), the number of main hyper-parameters or their ranges is higher than other ML models, which leads to larger search space scales, making them the most challenging for tuning [25]. Furthermore, since different ML algorithms have different hyper-parameter types(continuous, integer, and categorical), they should be treated differently in tuning processes [30]. For the ML models selected to explore in this study, ABC is chosen for the ML models to explore in this study since it enables parallel executions to improve tuning time, particularly for models that often require massive training time. Some other techniques, such as the G.A. approach, usually take more time than $\mathrm{ABC}$ since it is challenging to parallelize. Also, although techniques such as PSO have a reasonable convergence rate, they have a higher chance of sticking into local optimums and proved to be less efficient in comparison to $\mathrm{ABC}$ regarding performance [31].

\section{B. ABC Applications}

This section covers some of the previous studies of $\mathrm{ABC}$ for different optimization problems. One of the applications of $\mathrm{ABC}$ can be seen in [34] where authors proposed an ABCbased approach called IFAB, distinguishing between clean and unclean images. The authors modified the original $\mathrm{ABC}$ to work for discrete problems. Moreover, Sarac Essiz and Oturakci developed a comparative analysis to explore the impacts of the ABC-based feature selection algorithm [35] and eliminate non-informative features in the cyberbully detection problem. They also showed that their method has a better performance than some conventional methods like information gain, relief, and chi-square [35].

Amar and Zeraibi [36], combined ABC and Support Vector Regression (SVR) to predict minimum miscibility pressure and improve the oil recovery process. In their study, ABC was used to find the best hyper-parameters of the SVR model. In another study, Dokeroglu et al. developed a hybrid ABC optimization algorithm for the quadratic assignment problem using Tabu search to simulate exploration and exploitation phases [37]. They showed that $\mathrm{ABC}$ performs well for most quadratic assignment problems and can compete with other state-of-theart meta-heuristic algorithms existing in the literature.

Choong et al. utilized a modified choice function for the $\mathrm{ABC}$ algorithm. This algorithm adjusts the neighborhood search of the employed and onlooker phases. The results showed that the modified choice function brought advantages to the search process [38]. Since training ML models is timeconsuming, it is being argued that the use of algorithms such as the $\mathrm{ABC}$ algorithm is of great benefit to solve problems 
TABLE I: Comparison of GA, PSO, and ABC algorithms

\begin{tabular}{|c|c|c|c|}
\hline Method & Advantages & Disadvantages & Time Complexity \\
\hline GA & - No proper initialization is needed & $\begin{array}{l}\cdot \text { Poor with parallelization } \\
\text { - Introduces more hyper-parameters than PSO/ABC } \\
\bullet \text { Slow Convergence }\end{array}$ & $O\left(n^{2}\right)[32]$ \\
\hline PSO & $\begin{array}{c}\text { - Enables parallelization } \\
\text { - Faster convergence than GA or ABC }\end{array}$ & $\begin{array}{l}\text { - Needs proper initialization } \\
\text { - May stuck in local optimum }\end{array}$ & $O(n \log n)[6]$ \\
\hline $\mathbf{A B C}$ & $\begin{array}{c}\text { - Enables Parallelization } \\
\cdot \text { Higher efficiency } \\
\cdot \text { Balances exploration \& exploitation }\end{array}$ & $\begin{array}{l}\text { - Need proper initialization } \\
\text { - Slower convergence than PSO }\end{array}$ & $O(n \times D)[33]$ \\
\hline
\end{tabular}

caused by the Curse of Dimensionality (CoD) [39]. In [40], the authors proposed two algorithms to modify the original $\mathrm{ABC}$. The first algorithm employed neural network initialization, and the second algorithm utilized stochastic gradient descent in the employed phase of $\mathrm{ABC}$ to improve the convergence rate and observed promising results.

In another study, Zhao et al. performed a comparative analysis that proposed a modified version of $\mathrm{ABC}$ to improve the performance of SVM classification using parameter optimization. The proposed method utilized chaotic sequences for the initialization step, and they also defined an adaptive step size for the neighborhood search to boost the algorithm convergence. The modified $\mathrm{ABC}$ algorithm was examined to perform classification on two hyperspectral images. The authors then compared the results with three other PBAs and observed the superiority of the ABC method over the rest of the algorithms [41].

Pandiri and Singh [42] developed a hyper-heuristic-based $\mathrm{ABC}$ algorithm for the k-Interconnected multi-depot multitraveling salesman problem (k-IMDMTSP). The authors stated that due to the parameter values, it is impossible to have a unique algorithm that outperforms the rest of the algorithms emanating from k-IMDMTSP. Hence, they leveraged a hyperheuristic-based $\mathrm{ABC}$ algorithm for this problem. The authors presented an encoding scheme to be used inside the algorithm. The experimental results exhibited smaller search space and improved performance compared to other approaches existing in the literature.

In another study, Mazini et al. took advantage of the hyperparameters regulation method of $\mathrm{ABC}$ for feature selection to propose a reliable hybrid method to detect anomaly networkbased intrusion. Experimental results on a network traffic dataset exhibited improved performance and detection rate compared to other intrusion detection systems in various scenarios [43]. Gunel and Gor developed an ABC-based algorithm to solve initial value problems. To define a mutation operator, the authors used a dynamically constructed hypersphere to generate new food solutions to enhance the exploitation capability of the ABC. In this approach, the solutions for the differential equations were yielded through training neural networks [44].

In [45], Sayed et al. used an ABC-based approach to tune the hyper-parameter of SVM to improve the learning performance. The proposed method was compared with popular swarm algorithms and showed promising results. Agrawal also proposed an extended version of $\mathrm{ABC}$ using some features of the Gaussian $\mathrm{ABC}$ scheme to tackle some of the disad- vantages of the original $A B C$, such as a slow convergence. The proposed method was used to adjust the exploration and exploitation phases. They examined the proposed approach on some datasets and observed that the proposed method outperformed the original $\mathrm{ABC}$ in most experiments [46].

\section{HyPER-PARAMETER Optimization USING ABC}

There are different optimization problems in multiple fields. To such problems, there exist classical approaches and heuristic approaches. Classical techniques are not efficient enough in solving optimization problems, and this is primarily due to dimensionality. Using brute-force search, looking for the best hyper-parameter in the search space is an NP-hard problem with a time-complexity of $O\left(n^{k}\right)$ (where $\mathrm{k}$ is the number of hyper-parameters, $\mathrm{n}$ is the number of distinct values for each hyper-parameter). Heuristic approaches such as genetic and evolutionary algorithms do not suffer from many drawbacks of classical methods when dealing with large search spaces caused by $\mathrm{CoD}$. Among heuristics, $\mathrm{ABC}$ has shown more encouraging behavior. $\mathrm{ABC}$ is a population-based optimization technique and belongs to the family of stochastic SI methods. $\mathrm{ABC}$ is inspired by social interactions in honeybees and has recently garnered much success in different applications. It is as simple as some other swarm techniques such as PSO and is beneficial for NP-hard problems. Previous research shows that the $\mathrm{ABC}$ algorithm delivers relatively superior performance than PSO [31]. Hence, it is being explored in this study to find the close to the optimal set of hyper-parameters in ML algorithms.

\section{A. Time Complexity}

Table I summarizes GA, PSO, and ABC's advantages and disadvantages along with their time complexity. $\mathrm{ABC}$ requires a one-pass scan for each of the initialization, employed, and onlooker phases. Also, the ABC algorithm can repeat based on a maximum number of iterations defined for the algorithm. The time complexity for each of the neighborhood searches is $O(N * D)$ where $\mathrm{N}$ is the population size (number of solutions, and $D$ is the dimensionality. Therefore, the complexity of $\mathrm{ABC}$ can be presented as $O\left(3 N * D * I_{\max }\right)$ and $I_{\max }$ is the fixed maximum number of iterations. Therefore the time complexity of the $\mathrm{ABC}$ is $(O(N \times D))$.

\section{B. Advantages of $A B C$}

BO [29], GA [18] and PSO [47] are among the common approaches used for HPO problems. There are various features 
that $\mathrm{ABC}$ has which make it efficient in solving optimization problems, especially for hyper-parameter tuning are as follows:

1) The underlying concept is easy to understand and implementation offer high accuracy.

2) Although almost all meta-heuristic make use of randomization to have global research as well as local search, $\mathrm{ABC}$ balances the exploration and exploitation steps (local and global search) [28]. This enables the algorithm to search for various parts of the search area. Randomization also helps the model not get stuck in the local minimum and complete the global search. This is despite BO or PSO methods that may stick to a local optimum and fail to get to a global optimum [6].

3) Parallelization is one the advantages of PBA because each population can be assessed on one machine [25], while sequential methods such as BOs and GAs are challenging to parallelize since solutions are dependant on each other [30].

4) Some PBA methods such as GA have some additional hyper-parameters (number of generations, crossover, mutation, population size, and selection operators) to tune; therefore, GA has lower convergence speed [18]. Having fewer hyper-parameters to be tuned by the user is one of the advantages of methods such as PSO and $\mathrm{ABC}$.

\section{Fit ABC to HPO Problems}

In many optimization problems, including ML hyperparameter tuning, some of the hyper-parameters are discrete. Therefore, the vectors of hyper-parameters could consist of discrete (integer and categorical) or continuous (float) values. Since the basic ABC is only applicable to continuous problems, proper strategies should be adopted to apply them to discrete or combinatory problems. Hence, the first step to modifying the original $\mathrm{ABC}$ to adapt to the HPO problems is to modify the initial population generation. Each sample should be described as a vector, including a set of hyperparameters. Corresponding to each vector, there is a value for the objective function produced after training the ML model (model performance) and a fitness function calculated based on the objective function. After the population is generated, each vector is taken by one employed bee where it generates a new vector of hyper-parameters in the neighborhood, meaning that only one of the hyper-parameter in the current vector gets replaced with the same hyper-parameter of another vector. If the new vector has a better fitness, it gets replaced with the previous vector.

In the mutation step, onlooker bees start calculating the quality of the new population. Each of the vectors may or may not get selected depending on the computed quality. If a vector is selected, the onlooker bee generates a new vector of hyperparameters in the vicinity and assesses it by comparing it with the previous one. Similar to the employed bee phase, the better food source gets replaced. Finally, an exhausted vector of hyper-parameters (the vector that had the chance to improve the fitness but did not) is replaced with a random vector generated by the scout bee. Unlike the vectors generated in employed and onlooker phases, the new vector changes all the hyper-parameters (hence, not in the vicinity). This phase helps the algorithm not to stick to the local optimum. The above phases repeat until a stopping criterion is met where the algorithm stops and returns the best performance achieved so far.

\section{HyP-ABC: Modified ABC Algorithm}

\section{A. Converting continuous $A B C$ to combinatory version}

The schematic objective of HyP-ABC is shown in Figure 2. As mentioned in the previous section, we face a combination of hyper-parameters types while original $\mathrm{ABC}$ works only with continuous problems. Also, the range of variables in basic $\mathrm{ABC}$ is the same for all the dimensions. Therefore, proper strategies should be adopted to make it applicable to HPO problems.

There are different strategies used for tackling discrete optimization in swarm optimization [38], [48]-[50]. Rounding off is one of the most common approaches to tackle discrete variables. In this approach, the integer and continuous variables are treated similarly during the optimization process.

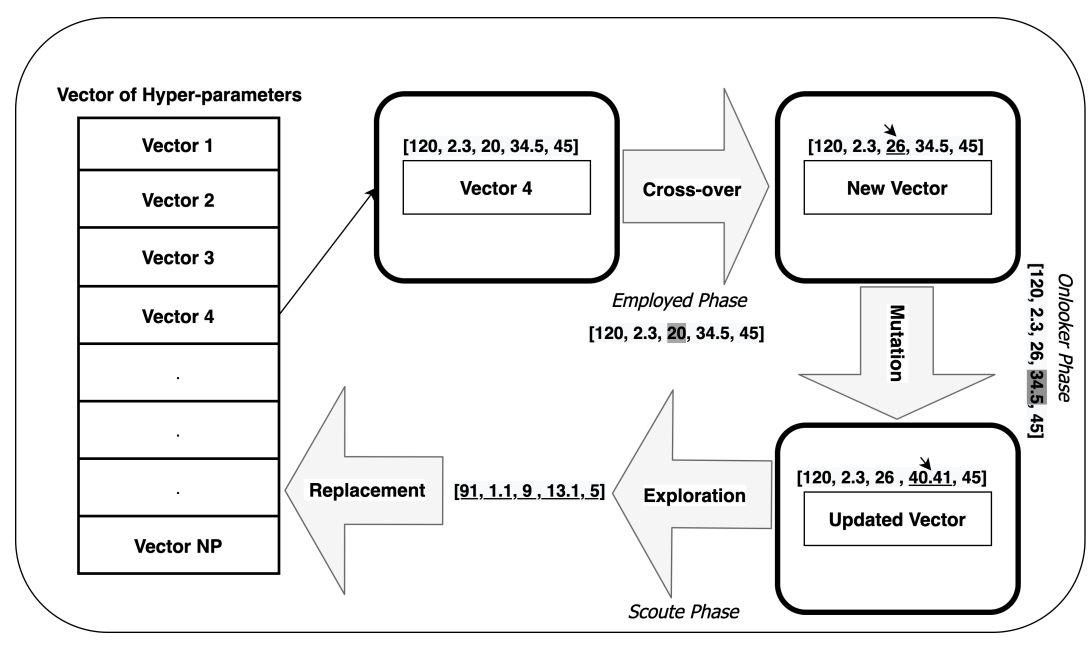

Fig. 2: General scheme of the HyP-ABC 
However, when the optimization process is done in most studies, the variables are rounded off to the closest integer number. Simplicity and low computational cost are among the main advantages of this method. However, entering impossible regions and high variation of fitness value of rounded value and the original value are among the disadvantages of this method. This study adjusts each vector's element based on its type and range in each iteration. Hence, the precise accuracy with the precise hyper-parameters is returned at the end of the optimization process. The remainder of this section explains how this process works.

As mentioned, the modified algorithm should treat various hyper-parameters differently in each iteration of generating and re-generating food sources. In this study, categorical variables in the initialization phase are encoded to integer numbers and treated as discrete variables afterward. Then, they are converted again to corresponding values after the optimization process is done and before training the model.

From this step on, if the selected hyper-parameter type by employed/onlooker is discrete, the newly generated food source is modified to an accepted vector within the defined range for that specific dimension. For continuous variables, the algorithm performs similarly to the original ABC. Algorithm 2 shows the detailed steps for HyP-ABC. As shown, another step is also added to check if the updated food source equals the current food source to prevent training the same vector of hyperparameter and save time. This is specifically useful when the selected hyper-parameter for an update is discrete (due to having fewer options and a higher chance of getting the same value after the update). For binary categorical variables (such as criterion in RF), we value flip the hyper-parameter value (flipped $=1$ - binary_value).

Values outside the ranges defined for the hyper-parameters in all phases get replaced with lower or higher bound values before training the ML model. The stopping condition in this algorithm is when the algorithm evaluates a specific number of evaluations.

\section{B. $H y P-A B C$ Steps}

For the random initialization of the population. Each solution is a vector of hyper-parameters, $X_{i}$, with the length of $D$, where $D$ is the dimension of vector or the number of hyperparameters. Unlike original $\mathrm{ABC}$, in $\mathrm{HyP}-\mathrm{ABC}$, each vector member may have discrete or continuous type with different define ranges:

$$
X_{i, j}=x_{m i n, j}+\operatorname{rand}(0,1)\left(x_{\max , j}-x_{m i n, j}\right)
$$

Where $X_{i, j}$ is the hyper-parameter value of food source $i$ in dimension $j$, and $x_{\max , j}$ and $x_{m i n, j}$ are upper-bound and lower-bound of $j_{t h}$ hyper-parameter. Then, each vector is assigned to an employed bee. The employed bee generate a new food source $N_{i, j}$ in that neighborhood by changing one of the hyper-parameters using below formula:

$$
N_{i, j}=x_{i, j}+\operatorname{rand}(-1,1)\left(x_{i, j}-x_{k, j}\right)
$$

Where $x_{k, j}$ represents the corresponding hyper-parameter value in the neighborhood. Then the fitness of the $N_{i}$ and
$X_{i}$ are compared and the better food source becomes a new member of the population afterward. Fitness is calculated from the objective function:

$$
f_{i t} t_{i}= \begin{cases}\frac{1}{1+f_{i}}, & f_{i} \geq 0 \\ 1+a b s\left(f_{i}\right), & f_{i}<0\end{cases}
$$

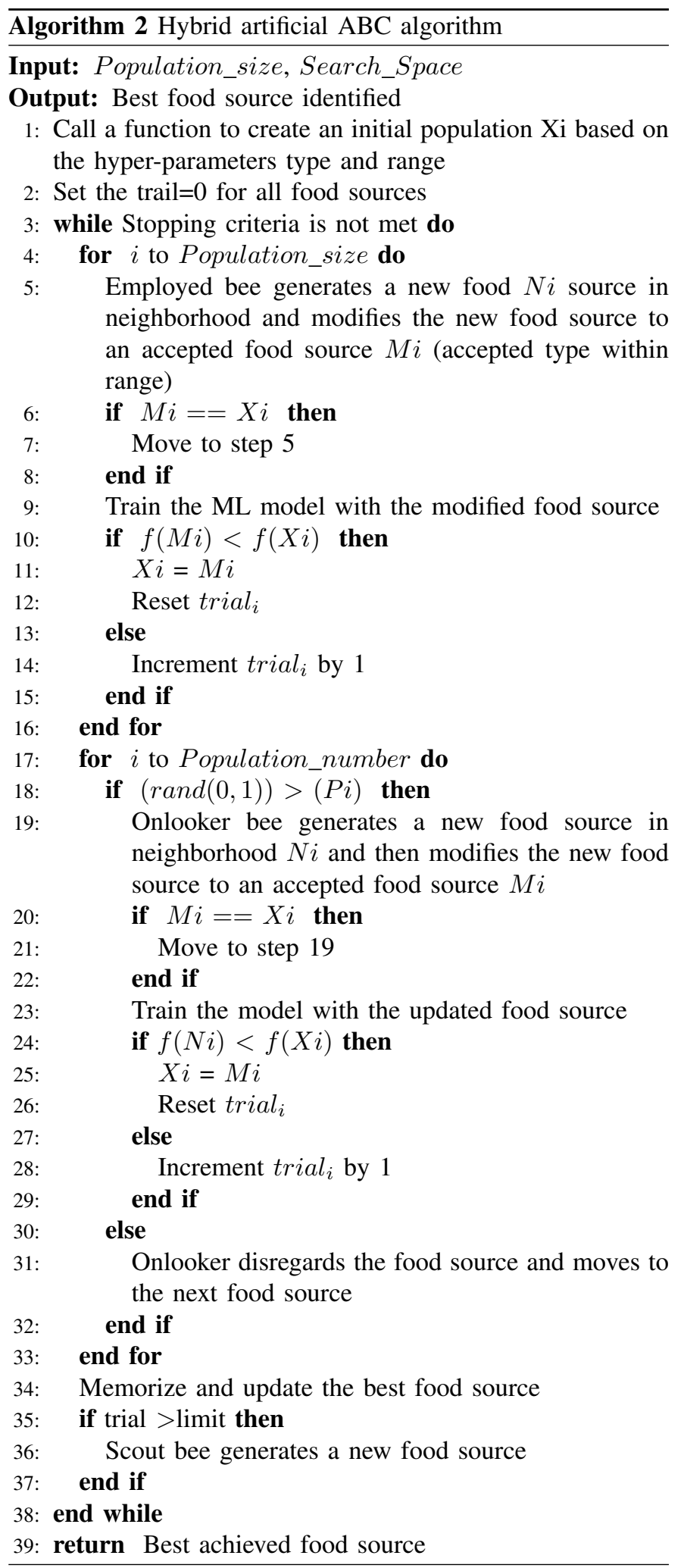




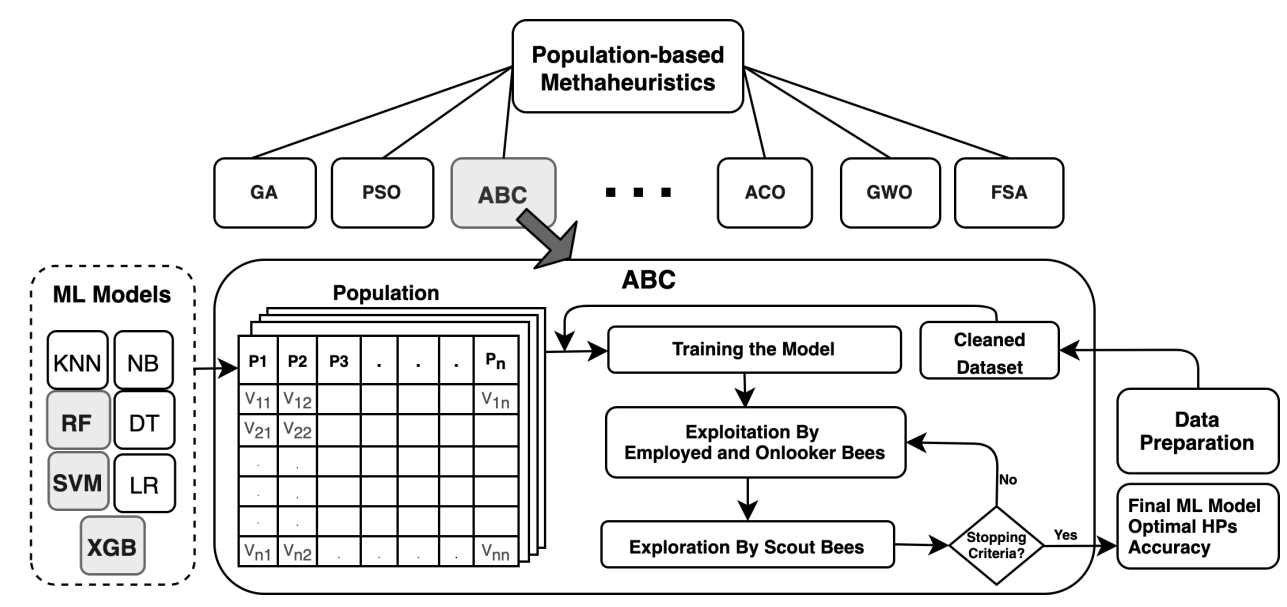

Fig. 3: The schematic process of the study

When employed bees are done with their part, they share the information with onlooker bees. Based on the received information they decide whether to further exploit a vector or not. In this phase, roulette wheel selection is utilized to calculate the probabilities, as shown below:

$$
P_{i}=\frac{f_{i}}{\sum_{j=1}^{P N}\left(f_{j}\right)}
$$

Where $f_{i}$ is the fitness value for the $i$ th food source and $P N$ is the population size. Next, scout bees start exploring random configurations in search space without using experience or memorizing the locations. They do not use greedy selection when exploring new food sources. Exploration by scout bees is through the below formula:

$$
X_{i, j}=x_{\min }+\operatorname{rand}(0,1)\left(x_{\max }-x_{\min }\right)
$$

\section{EXPERIMENTAL Methodology}

In this section, we describe the methodology used in this study aiming to improve the efficiency of a recent work in 2021, using conventional HPO methods [11]. The process is performed in several consecutive phases, and it includes data pre-processing, feature engineering, leveraging ML models, and $\mathrm{ABC}$-based optimization steps.

\section{A. Data pre-processing and feature Engineering}

Data pre-processing is a technique to transform the raw data into an understandable format for ML algorithms. It includes data cleansing, data normalization, and data reduction. For data cleansing, we remove the features with more than $60 \%$ percent of the values missing. Data normalization is also a method to standardize the data when the different features' values vary widely. StandardScaler from Scikit-learn library was used for scaling the input data. We filter the data in the data reduction step and included only the target population, students from computing fields. One-hot encoding (categorical encoding variables to binary variables for each unique category) is also used in the feature engineering step.

\section{B. Hyper-parameter tuning}

Tuning hyper-parameters is essential to yielding the best performance of an ML model. Due to the varied nature of the hyper-parameters in different ML models, we implement a HyP-ABC to tune the hyper-parameters of three ML models. HyP-ABC is replaced with automated HPO methods performed in [11]. Algorithm 3 shows the general steps of HyP$\mathrm{ABC}$ amd Figure 3 shows the schematic process of HyP-ABC framework.

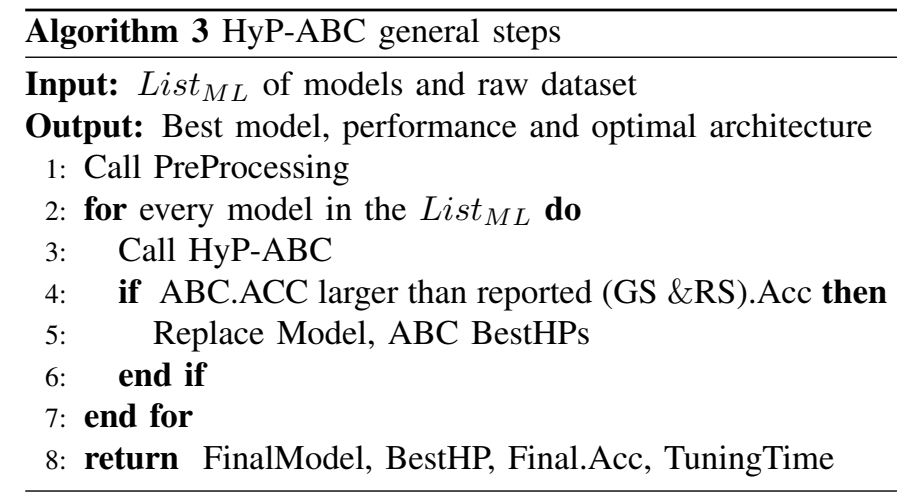

Since RF, XGBoost, and SVM are computationally expensive when using automated HPO methods [11], they are selected to be explored in this study. The tuning time complexity in these models is mainly due to the model's complexity or the ranges of hyper-parameters.

\section{EXPERIMENTAL RESULTS}

In this section, we discuss the dataset, evaluation metrics, and experimental results in depth.

\section{A. Dataset}

In this study, we use a real-world educational data, MultipleInstitution Database for Investigating Engineering Longitudinal Development (MIDFIELD) [51], to predict students' success in computing majors. This dataset is a unit-record longitudinal database for all undergraduate students from 19 universities across the United States. MIDFIELD contains all 


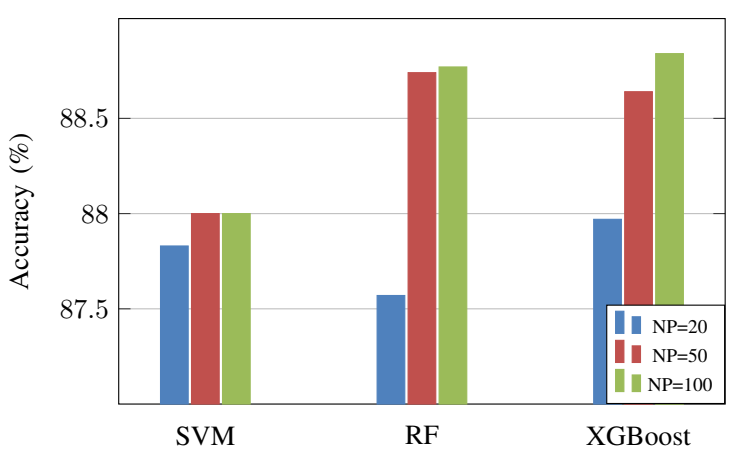

(a) Cross Validation

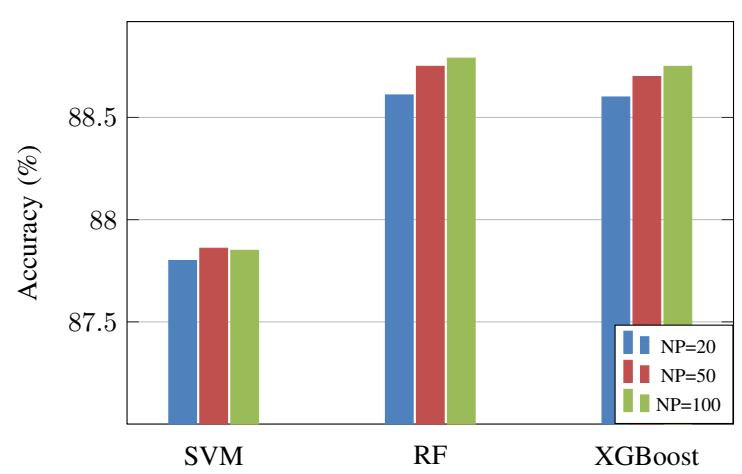

(b) 80:20 Ratio Test/Train Set

Fig. 4: Effect of number of food sources on accuracy after specific number of iterations

the information that shows on students' academic records, such as demographic data and information about field, enrollment, graduation, and their academic performance before and during school. We used a reduced version of MIDFIELD, including only students majoring in computing fields (with $\mathrm{CIP}=11$ ). This version of the MIDFIELD dataset is used for a binary classification problem and has 4532 samples with 91 features.

\section{B. Metrics for Performance Evaluation}

The metrics used in this experiment to assess the performance of the classification and the proposed framework is accuracy. Accuracy is the fraction of the number of correct predictions to the total number of predictions. This metric is used since the dataset is balanced and the number of samples belonging to each class is almost equal(46:54).

Cross-validation $(\mathrm{CV})$ is used to reduce or prevent over-fitting. $\mathrm{CV}$ helps finding a stable optimum that is suitable for all the subsets of the dataset instead of only a particular validation set [25]. It is important to note that CV also increases the tuning time by the number of folds. Therefore, we used 3-fold crossvalidation to avoid high tuning times. However, we paralleled the $\mathrm{CV}$ process to reduce the impact of $\mathrm{CV}$ on execution time. We also separated the MIDFIELD dataset into train and test sets and repeated the experiment. The train set contains $80 \%$ of the dataset, and the test set includes $20 \%$ of the dataset.

In this experiment, the Scikit-learn and XGBoost libraries were used to leverage ML models. All experiments were conducted using Python 3.7 on AWS servers with four v-CPUs up to a 3.0 $\mathrm{GHz}$ scalable processor and $16 \mathrm{~GB}$ RAM.
TABLE III: Comparison of Time Executions having a desirable accuracy as stopping point applying HyP-ABC

\begin{tabular}{|c|c|c|c|}
\hline \multicolumn{4}{|c|}{ 80:20 ratio Test-Train set } \\
\hline & \multicolumn{3}{|c|}{ Tuning Time (hrs) } \\
\hline Population size (N) & $\mathbf{2 0}$ & $\mathbf{5 0}$ & $\mathbf{1 0 0}$ \\
\hline SVM & 23.95 & 53.05 & 62.57 \\
\hline RF & 2.37 & 9.07 & 3.18 \\
\hline XGBoost & 4.07 & $\mathbf{5 . 8 5}$ & 4.23 \\
\hline \hline With 3-Fold Cross Validation $(N=50)$ \\
\hline SVM & \multicolumn{3}{|c|}{34.15} \\
\hline RF & \multicolumn{3}{|c|}{29.55} \\
\hline XGBoost & \multicolumn{3}{|c|}{} \\
\hline
\end{tabular}

\section{Results}

HyP-ABC is developed to achieve the optimal or close to the optimal set of hyper-parameters of SVM, RF, and XGBoost algorithms. The higher number of hyper-parameters with possible wide ranges or complexity and the model's objective function are the motivation of selecting these ML algorithms. Figure 4(a) and 4(b) show the effect of populations size on accuracy among the three ML models using $\mathrm{CV}$ as well as using train and test sets; As can be seen, the larger the population size, the better the accuracy. However, it shows that the impact of population size decreases when it gets very large (large populations also have the disadvantage of increasing the execution time). Our experiment considers the population size as 50 for comparisons with other state-of-the-art HPO

TABLE II: Performance evaluation of utilizing HPO methods to the ML classifiers on the MIDFIELD dataset $(\mathrm{CV}=3)$

\begin{tabular}{|c|c|c|c|}
\hline & \multicolumn{3}{|c|}{ Accuracy (\%) } \\
\hline HPO Method / ML Classifier & RF & XGBoost & SVM \\
\hline GS (minimized search space) & 86.24 & 86.33 & 85.38 \\
\hline RS & 87.37 & 86.90 & 86.03 \\
\hline HyperBand & 86.74 & 87.75 & 87.54 \\
\hline BO-GP & 88.71 & 88.71 & 87.91 \\
\hline BO-TPE & 88.33 & 88.73 & 87.90 \\
\hline PSO & 88.76 & 88.63 & 87.98 \\
\hline GA & 87.51 & 88.56 & $\mathbf{8 8 . 0 8}$ \\
\hline HyP-ABC & $\mathbf{8 8 . 7 7}$ & $\mathbf{8 8 . 8 4}$ & $\mathbf{8 8 . 0 0}$ \\
\hline
\end{tabular}


methods. Table III shows the comparison of execution time when setting a desirable accuracy as the stopping point. As can be seen from the table, SVM has the highest execution time due to the SVM model's complexity and expensive objective functions. Table II summarizes the results and shows the accuracy of different HPO methods among different ML models having the same resource (number of iterations). As shown, the accuracy of the HyP-ABC is equal to or more than the rest of the approaches, showing the algorithm's capability for tuning ML models hyper-parameters. It is important to note that for RF and XGBoost algorithms, the GS tuning time considering all the configurations leads to a tuning time of more than years. This estimation was calculated from the average tuning time for each configuration from the RS experiment. Hence, the experiment for the GS method is implemented using a reduced search space with larger steps to make the experiment feasible.

Unlike model-free methods such as GS and RS, HyP-ABC presented in this study is a model-based method. In other words it has a methodical way of moving toward the optimal solution. The results of the study show that among all the ML algorithms developed in this experiment, XGBoost had the best accuracy score. Hence, XGBoost is the final model to be used on the MIDFIELD dataset.

\section{CONCLUSION AND DiscusSiOnS}

In this paper, a modified evolutionary optimization algorithm for hyper-parameter tuning in ML models referred to as $\mathrm{HyP}-\mathrm{ABC}$ is proposed to enable hyper-parameter tuning of ML classifiers. We carried out the experiment using the MIDFIELD dataset. The behavior of HyP-ABC has been explored under a different number of populations, and the accuracy and execution time of the HyP-ABC has been compared with existing HPO methods in an earlier study.

Experimental results verified that HyP-ABC is competitive with other tuning approaches used in the state-of-theart methods, Hyp-ABC improves the classification accuracy, and more importantly, it decreases the tuning time. HyP$\mathrm{ABC}$ can be deployed to solve the HPO problems with large search spaces. To summarize, HyP-ABC is recommended for optimizing RF, XGBoost, and SVM. In future work, we will explore the semantic initialization of the population to improve the convergence rate further. Also, the time complexity of algorithms such as SVM is highly dependent on the number of features and samples of a dataset, and hence their function evaluation can be prolonged for large-scale datasets. Exploration of feature selection and data reduction techniques is encouraged for future work.

\section{ACKNOwledgment}

The authors acknowledge the University Graduate School (UGS), Florida International University for providing support for this research in the form of Dissertation Year Fellowships (DYF) for Mrs. Leila Zahedi.

\section{REFERENCES}

[1] F. G. Mohammadi, H. R. Arabnia, and M. H. Amini, "On parameter tuning in meta-learning for computer vision," in 2019 International Conference on Computational Science and Computational Intelligence (CSCI). IEEE, 2019, pp. 300-305.

[2] A. A. Neath and J. E. Cavanaugh, "The bayesian information criterion: background, derivation, and applications," Wiley Interdisciplinary Reviews: Computational Statistics, vol. 4, no. 2, pp. 199-203, 2012.

[3] A. Biem, "A model selection criterion for classification: Application to hmm topology optimization," in Seventh International Conference on Document Analysis and Recognition, 2003. Proceedings. IEEE, 2003. pp. 104-108.

[4] M. Claesen and B. De Moor, "Hyperparameter search in machine learning," arXiv preprint arXiv:1502.02127, 2015.

[5] P. Sharma, K. Chaudhary, and M. Khan, "The art-of-hyper-parameter optimization with desirable feature selection," Proceedings of 2021 International Conference on Medical Imaging and Computer-Aided Diagnosis (MICAD 2021), pp. 218-227, 2022.

[6] L. Yang and A. Shami, "On hyperparameter optimization of machine learning algorithms: Theory and practice," Neurocomputing, vol. 415, pp. 295-316, 2020.

[7] G. Sui and Y. Yu, "Bayesian contextual bandits for hyper parameter optimization," IEEE Access, vol. 8, pp. 42 971-42 979, 2020.

[8] S. Bhattacharyya, S. Majumder, P. Debnath, and M. Chanda, "Arrhythmic heartbeat classification using ensemble of random forest and support vector machine algorithm," IEEE Transactions on Artificial Intelligence, vol. 2, no. 3, pp. 260-268, 2021.

[9] V. M. Vu, A. Bibal, and B. Frénay, "Constraint preserving score for automatic hyperparameter tuning of dimensionality reduction methods for visualization," IEEE Transactions on Artificial Intelligence, vol. 2, no. 3, pp. 269-282, 2021

[10] H. Yin, K. Gai, and Z. Wang, "A classification algorithm based on ensemble feature selections for imbalanced-class dataset," in 2016 IEEE 2nd International Conference on Big Data Security on Cloud (BigDataSecurity), IEEE International Conference on High Performance and Smart Computing (HPSC), and IEEE International Conference on Intelligent Data and Security (IDS). IEEE, 2016, pp. 245-249.

[11] L. Zahedi, F. G. Mohammadi, S. Rezapour, M. W. Ohland, and M. H. Amini, "Search algorithms for automated hyper-parameter tuning," The 17th International Conference on Data Science (Accepted), 2021.

[12] F. G. Mohammadi, M. H. Amini, and H. R. Arabnia, "Applications of nature-inspired algorithms for dimension reduction: enabling efficient data analytics," in Optimization, Learning, and Control for Interdependent Complex Networks. Springer, 2020, pp. 67-84.

[13] H. Badem, A. Basturk, A. Caliskan, and M. E. Yuksel, "A new hybrid optimization method combining artificial bee colony and limitedmemory bfgs algorithms for efficient numerical optimization," Applied Soft Computing, vol. 70, pp. 826-844, 2018.

[14] T. Ozcan and A. Basturk, "Human action recognition with deep learning and structural optimization using a hybrid heuristic algorithm," Cluster Computing, pp. 1-14, 2020.

[15] _ , "Transfer learning-based convolutional neural networks with heuristic optimization for hand gesture recognition," Neural Computing and Applications, vol. 31, no. 12, pp. 8955-8970, 2019.

[16] D. Yazdani, R. Cheng, D. Yazdani, J. Branke, Y. Jin, and X. Yao, "A survey of evolutionary continuous dynamic optimization over two decades-part b," IEEE Transactions on Evolutionary Computation, vol. 25, no. 4, pp. 630-650, 2021.

[17] J. Pierezan and L. D. S. Coelho, "Coyote optimization algorithm: a new metaheuristic for global optimization problems," in 2018 IEEE congress on evolutionary computation (CEC). IEEE, 2018, pp. 1-8.

[18] S. Lessmann, R. Stahlbock, and S. F. Crone, "Optimizing hyperparameters of support vector machines by genetic algorithms." in IC-AI, 2005, pp. 74-82.

[19] Y. Shi, "Particle swarm optimization," IEEE connections, vol. 2, no. 1, pp. 8-13, 2004.

[20] V. Maniezzo, L. M. Gambardella, and F. De Luigi, "Ant colony optimization," in New Optimization Techniques in Engineering. Springer, 2004, pp. 101-121.

[21] S. Mirjalili, S. M. Mirjalili, and A. Lewis, "Grey wolf optimizer," Advances in engineering software, vol. 69, pp. 46-61, 2014.

[22] X.-1. Li, "An optimizing method based on autonomous animats: fishswarm algorithm," Systems Engineering-Theory \& Practice, vol. 22, no. 11 , pp. 32-38, 2002. 
[23] D. Karaboga, "An idea based on honey bee swarm for numerical optimization," Technical report-tr06, Erciyes university, engineering faculty, computer engineering department, Tech. Rep., 2005.

[24] Q. Yao, M. Wang, Y. Chen, W. Dai, Y.-F. Li, W.-W. Tu, Q. Yang, and Y. Yu, "Taking human out of learning applications: A survey on automated machine learning," arXiv preprint arXiv:1810.13306, 2018.

[25] F. Hutter, L. Kotthoff, and J. Vanschoren, Automated machine learning: methods, systems, challenges. Springer Nature, 2019.

[26] D. Karaboga, B. Akay, and C. Ozturk, "Artificial bee colony (abc) optimization algorithm for training feed-forward neural networks," in International conference on modeling decisions for artificial intelligence. Springer, 2007, pp. 318-329.

[27] D. Karaboga and B. Basturk, "A powerful and efficient algorithm for numerical function optimization: artificial bee colony (abc) algorithm," Journal of global optimization, vol. 39, no. 3, pp. 459-471, 2007.

[28] D. Karaboga and B. Akay, "A comparative study of artificial bee colony algorithm," Applied mathematics and computation, vol. 214, no. 1, pp. 108-132, 2009.

[29] K. Eggensperger, M. Feurer, F. Hutter, J. Bergstra, J. Snoek, H. Hoos, and K. Leyton-Brown, "Towards an empirical foundation for assessing bayesian optimization of hyperparameters," in NIPS workshop on Bayesian Optimization in Theory and Practice, vol. 10, 2013, p. 3.

[30] N. DeCastro-García, Á. L. Muñoz Castañeda, D. Escudero García, and M. V. Carriegos, "Effect of the sampling of a dataset in the hyperparameter optimization phase over the efficiency of a machine learning algorithm," Complexity, pp. 1-16, 2019.

[31] V. R. Kulkarni and V. Desai, "Abc and pso: A comparative analysis," in 2016 IEEE International Conference on Computational Intelligence and Computing Research (ICCIC). IEEE, 2016, pp. 1-7.

[32] S. Singh, N. Rajpal, and A. K. Sharma, "K-fault tolerant in mobile adhoc network under cost constraint," in 2011 3rd International Conference on Electronics Computer Technology, vol. 6. IEEE, 2011, pp. 368-372.

[33] S. Sharma and P. Bhambu, "Artificial bee colony algorithm: A survey," International Journal of Computer Applications, vol. 149, no. 4, pp. $11-19,2016$.

[34] F. G. Mohammadi and M. S. Abadeh, "Image steganalysis using a bee colony based feature selection algorithm," Engineering Applications of Artificial Intelligence, vol. 31, pp. 35-43, 2014.

[35] E. Sarac Essiz and M. Oturakci, "Artificial bee colony-based feature selection algorithm for cyberbullying," The Computer Journal, vol. 64, no. 3, pp. 305-313, 2021

[36] M. N. Amar and N. Zeraibi, "Application of hybrid support vector regression artificial bee colony for prediction of $\mathrm{mmp}$ in co2-eor process," Petroleum, vol. 6, no. 4, pp. 415-422, 2020.

[37] T. Dokeroglu, E. Sevinc, and A. Cosar, "Artificial bee colony optimization for the quadratic assignment problem," Applied soft computing, vol. 76, pp. 595-606, 2019.

[38] T. Chang, D. Kong, N. Hao, K. Xu, and G. Yang, "Solving the dynamic weapon target assignment problem by an improved artificial bee colony algorithm with heuristic factor initialization," Applied Soft Computing, vol. 70, pp. 845-863, 2018.

[39] E. Hancer, B. Xue, M. Zhang, D. Karaboga, and B. Akay, "Pareto front feature selection based on artificial bee colony optimization," Information Sciences, vol. 422, pp. 462-479, 2018.

[40] C. Mala, V. Deepak, S. Prakash, and S. L. Srinivasan, "A hybrid artificial bee colony algorithmic approach for classification using neural networks," in 2nd EAI International Conference on Big Data Innovation for Sustainable Cognitive Computing. Springer, 2021, pp. 339-359.

[41] C. Zhao, H. Zhao, G. Wang, and H. Chen, "Improvement svm classification performance of hyperspectral image using chaotic sequences in artificial bee colony," IEEE Access, vol. 8, pp. 73 947-73 956, 2020.

[42] V. Pandiri and A. Singh, "A hyper-heuristic based artificial bee colony algorithm for k-interconnected multi-depot multi-traveling salesman problem," Information Sciences, vol. 463, pp. 261-281, 2018.

[43] M. Mazini, B. Shirazi, and I. Mahdavi, "Anomaly network-based intrusion detection system using a reliable hybrid artificial bee colony and adaboost algorithms," Journal of King Saud University-Computer and Information Sciences, vol. 31, no. 4, pp. 541-553, 2019.

[44] K. Günel and I. Gör, "A modification of artificial bee colony algorithm for solving initial value problems," TWMS Journal of Applied and Engineering Mathematics, vol. 9, no. 4, pp. 810-821, 2019.

[45] G. I. Sayed, M. Soliman, and A. E. Hassanien, "Parameters optimization of support vector machine based on the optimal foraging theory," in Machine Learning Paradigms: Theory and Application. Springer, 2019, pp. 309-326.
[46] S. Agrawal et al., "Modified gbest-guided abc algorithm approach applied to various nonlinear problems," in Optical and Wireless Technologies. Springer, 2020, pp. 615-623.

[47] X. Guo, J. Yang, C. Wu, C. Wang, and Y. Liang, "A novel lssvms hyper-parameter selection based on particle swarm optimization," Neurocomputing, vol. 71, no. 16-18, pp. 3211-3215, 2008.

[48] I. Ziari, G. Ledwich, A. Ghosh, and G. Platt, "Integrated distribution systems planning to improve reliability under load growth," IEEE transactions on Power Delivery, vol. 27, no. 2, pp. 757-765, 2012.

[49] W.-C. Wu and M.-S. Tsai, "Feeder reconfiguration using binary coding particle swarm optimization," International Journal of Control, Automation, and Systems, vol. 6, no. 4, pp. 488-494, 2008.

[50] R. Durgut, H. Kutucu, and S. Akleylek, "An artificial bee colony algorithm for solving the weapon target assignment problem," in Proceedings of the 7th International Conference on Information Communication and Management, 2017, pp. 28-31.

[51] M. Ohland, G. Zhang, B. Thorndyke, and T. J. Anderson, "The creation of the multiple institution database for investigating engineering longitudinal development (midfield)," in 2004 Annual Conference, 2004, pp. $9-1244$.

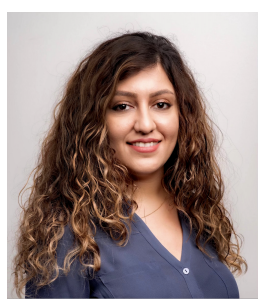

Leila Zahedi received her B.S. degree in computer engineering from the University of Isfahan, Iran, and her M.S. degree in computer science from Florida International University (FIU), Miami, Fl, US. She is currently pursuing a Ph.D. degree in computer science at Florida International University, Knight Foundation School of Computing and Information Sciences (KFCIS), FL, USA. She has published around 15 journal and conference papers. She is currently a Research Assistant with the Sustainability, Optimization, and Learning for InterDependent networks (SOLID) laboratory at FIU, Miami, FL, U.S. Her research interest includes the development of efficient optimization algorithms for automated machine learning, data science, and educational data mining.

Mrs. Zahedi's awards and honors include six Scholarships to attend the Grace Hopper and NSF TAPIA conference, among others. She was also the invited speaker at NSF Re-Enter STEM through Emerging Technology (RESET) Conference.

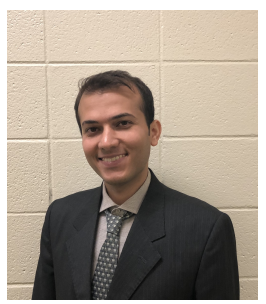

Farid Ghareh Mohammadi is currently a Ph.D candidate of Computer Science at University of Georgia, USA. He received his M.Sc. degree from Tarbiat Modares University in 2013, and a B.Sc. degree from Payamnoor University in 2011. His research spans on machine learning, optimization, evolutionary and nature-inspired algorithms, and computer vision. He has published more than 20 journal and conference papers, and book chapters.

Mr.Ghareh Mohammadi was selected as an Outstanding Reviewer in Journal of Visual Communication and Image Representation in 2017. He served as conference chair and was chosen as Outstanding Achievement Award in the field of Deep learning at CSCI'19, Las Vegas, USA. He won the 2019 POOIA Outstanding Achievement Award and The POOIA Student Scholarship Award in recognition of sustained record of academic excellence. (Homepage: www.faridghm.com)

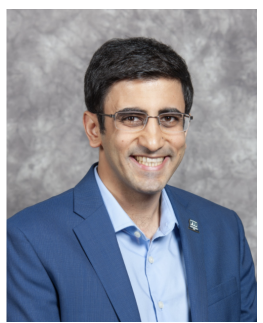

M. Hadi Amini (GSM'11, M'19) is an Assistant Professor at KFCIS at FIU. He is the director of SOLID laboratory (www.solidlab.network). He received his Ph.D. in Electrical and Computer Engineering from Carnegie Mellon University in 2019, where he received his M.Sc. degree in 2015. He also holds a doctoral degree in Computer Science and Technology. Prior to that, he received M.Sc. degree from Tarbiat Modares University in 2013, and the B.Sc. degree from Sharif University of Technology in 2011

Dr.Amini's research interests include distributed optimization and learning algorithms, distributed computing and intelligence, sensor networks, interdependent networks, and cyber-physical-social resilience. Application domains include smart cities, energy systems, transportation electrification, and healthcare. (Homepage: www.hadiamini.com) 